\section{Nuclear Disintegrations caused by Fast Cosmic-Ray Neutrons in Photographic Emulsions}

$O_{N}$ the same plates as those described in my previous communication ${ }^{1}$, I have studied the relation between the single tracks and the stars, and tried to elucidate the question of their origin. A detailed analysis of 600 stars and of 3,000 single tracks was carried out. My measurements support the assumption that the single tracks are caused mainly by fast neutrons in glancing collision with the nuclei of the emulsion. From the topological distribution of the stars on the plates and from the angular and energy distributions of their prongs (protons, $\alpha$-particles and recoil tracks), I have shown, applying the results of the evaporation theory ${ }^{2}$, that the great majority of the stars are also caused by fast neutrons in glancing collision without the capture of the incident neutrons in the nuclei. 'The energy distribution of fast protons emitted from stars was used to determine the range of nuclear forces following E. Bagge ${ }^{3}$ and G. Ortner ${ }^{4}$. From my measurements $I$ obtained the value $x^{-1}=(2 \cdot 6 \pm 0 \cdot 4) \times 10^{-13} \mathrm{~cm}$.

Details of this work will be published in the Bulletin international de l'Académie tcheque des Sciences. I wish to express my gratitude to Prof. $V$. Petržilka for helpful advice in this work.

Institute of Nuclear Physics,

J. Pernegr

Academy of Sciences, and

Institute of Physics,

Charles' University,

Prague.

July 25.

1 Pernegr, J. (see preceding communication).

- Le Couteur, K. J., Proc. Phys. Soc, A, 63, 259 (1950).

- Bagge, E., Phys. Z., \21, 461 (1944).

- Ortner, G., Ber._Akad.'Wien, 149, 259 (1940).

\section{Transition Effect of the Star-producing Radiation}

To prove the existence of the transition effect of the star-producing radiation suggested by G. Bernardini' ${ }^{1}$, an experiment relating to this question was carried out in the High Tatras in the winter of 1950. Six batches of Ilford $C 2$ plates with emulsion thickness of $200 \mu$ were exposed during 45 days at Lomnický Stít $(2,640 \mathrm{~m}$.). One batch of plates was exposed without any absorber, while the others were placed under different layers of lead of increasing thicknesses from $1 \mathrm{~cm}$. to $5 \mathrm{~cm}$.

The experimental results concerning the numbers of stars observed in the same volumes of the emulsion under different thicknesses of the absorber are plotted in the accompanying graph. Comparison of the experimental values with the postulated exponential absorption curve shows a well-pronounced maximum of the frequency of stars under the layer of about $20 \mathrm{gm} . / \mathrm{cm} .^{2}$ lead. The dotted exponential line was drawn using the value for the absorption coefficient $\lambda^{b}=310 \mathrm{gm} . / \mathrm{cm}^{2}$.

Further observations are being collected, and definite measurements with possible interpretations of this effect will be published later in the Bulletin international de l'Académie tchèque des Sciences.

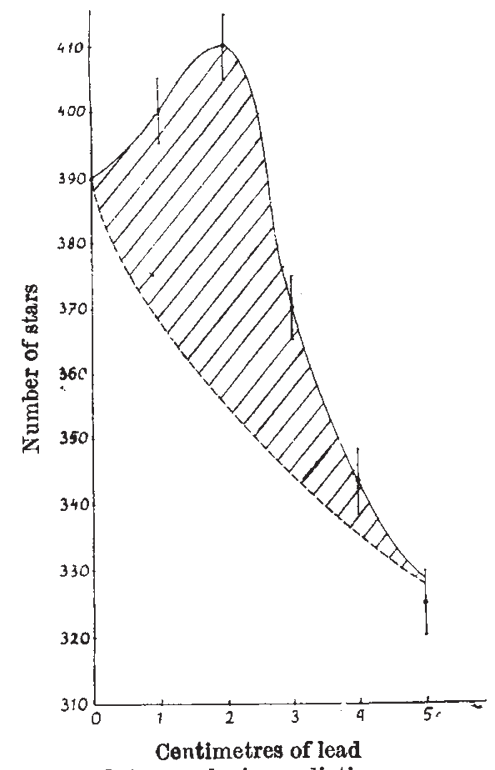

Transition effect of star-producing radiation : ....., theoretical exponential absorption;

I wish to thank Dr. J. Pernegr for suggestions and help in this work.

Note added in proof. Since this communication was written, G. Belliboni, L. Fabrichesi, L. de Marco and M. Merlin ${ }^{2}$ have published a paper in which they describe measurements similar to those $I$ have made.

Institute of Nuclear Physics,

L. TOMÁ̌̌KKOVA

Academy of Sciences, and

Institute of Physics,

Charles' University, Prague.

July 25.

'Bernardini, G., "Cosmic Radiation", 21, in the Colston Papers (Butterworths Scientific Publications, London, 1949).

2 Nuovs Cimento, 8, 374 (1951).

\section{The Decay Scheme of Zinc-65}

According to a short report given by Good and Peacock $^{1}$, the radioactive isotope zinc-65 decays 97.8 per cent by $K$-capture and $2 \cdot 2$ per cent by positron emission. They also found that 54 per cent of the capture processes go directly to the ground-state of the product nucleus copper-65, while 46 per cent go to the $1 \cdot 11-\mathrm{MeV}$. excited state.

In the present work, the branching ratio between the two capture processes has been investigated, using a selective method for the detection of the $\mathrm{X}$-rays in addition to $\mathrm{X}-\boldsymbol{\gamma}$ coincidences and $\mathbf{a}$ calibrated $\gamma$-ray counter.

The source used was a thin layer of finely ground zinc-65 spread on a 'Cellophane' foil and covered with a few drops of a diluted solution of cellulose. This source was placed between two cylindrical GeigerMüller counters - an ordinary $\gamma$-ray counter with l-mm. thick brass walls, and an X-ray counter with aluminium walls $0.05 \mathrm{~mm}$. thick.

Let $K_{1}$ be the number of capture processes going to the ground-state and $K_{2}$ the number going to the excited state. Then a branching ratio $p$ can be defined as : 